\section{Emergent Sensing of Complex Environments by Mobile Animal Groups}

\author{
Andrew Berdahl, ${ }^{{ }^{*}}+$ Colin ]. Torney, ${ }^{1 *}$ Christos C. Ioannou,,${ }^{1,2}$ jolyon J. Faria, ${ }^{1}$ Iain D. Couzin ${ }^{1} \dagger$
}

The capacity for groups to exhibit collective intelligence is an often-cited advantage of group living. Previous studies have shown that social organisms frequently benefit from pooling imperfect individual estimates. However, in principle, collective intelligence may also emerge from interactions between individuals, rather than from the enhancement of personal estimates. Here, we reveal that this emergent problem solving is the predominant mechanism by which a mobile animal group responds to complex environmental gradients. Robust collective sensing arises at the group level from individuals modulating their speed in response to local, scalar, measurements of light and through social interaction with others. This distributed sensing requires only rudimentary cognition and thus could be widespread across biological taxa, in addition to being appropriate and cost-effective for robotic agents.

$\mathrm{R}$ esearch on collective intelligence has demonstrated how organisms, including humans, can improve decision-making accuracy by appropriately pooling individual estimates (1-8). In the earliest study, in 1907, Sir Francis Galton made a near-perfect estimate of the weight of an ox by using 787 guesses made by others (9). Pooling of information is not exclusive to the human domain; for example, it has also been suggested that aggregating imperfect esti-

${ }^{1}$ Department of Ecology and Evolutionary Biology, Princeton University, Princeton, N] 08544, USA. ${ }^{2}$ School of Biological Sciences, University of Bristol, Woodland Road, Bristol BS8 IUG, UK.

*These authors contributed equally to this work.

†To whom correspondence should be addressed. E-mail: aberdahl@princeton.edu (A.B.); icouzin@princeton.edu (I.D.C.) mates may help organisms, or cells, navigate weak or noisy environmental gradients (2-5). If each individual makes an error-prone estimate of the local gradient, it may benefit by also basing its movement decisions on the direction of motion of others, termed the "many wrongs" hypothesis (3).

Despite the importance of collective sensing to the ecology of many social species $(10,11)$ and the value to bio-inspired technological applications [such as particle swarm optimization (12), or swarm robotics (13)], with the exception of the social insects (8), we do not know how grouping enhances sensing capabilities in animal groups. Here, we use an integrated experimental and theoretical approach to address this deficit. We use schooling fish (golden shiners, Notemigonus crysoleucas) as our model experimental system and take advantage of their natural preference for a shaded (darker) habitat (14). Thus, our ex- periments do not require training and are not susceptible to confounding factors relating to competition for, or consumption of, a preferred resource. Shiners school naturally in shallow water (15) and remain highly cohesive (14), which allowed us to explore the role of group size during a gradient detection task.

Our experiments were conducted with juvenile fish (body length $4.9 \pm 0.5 \mathrm{~cm}$ ) in a shallow tank ( $213 \mathrm{~cm}$ by $122 \mathrm{~cm}, 8$-cm water depth). Dynamic light fields were projected onto the tank. These fields consisted of a circular patch that was darkest at its center and transitioned smoothly (as an exponential) to the brightest light levels. Noise was added to this gradient to generate local variability in space and time. Furthermore, the circular patch itself moved at a constant speed between a series of randomly selected locations within the tank. See (10) for further details. The task for the fish was to track the preferred, darker regions of this dynamic environment.

We investigated the performance of single fish and groups of $2,4,8,16,32,64,128$, and 256 individuals. Three levels of environmental noise were employed. For the lowest value, the light field was dominated by the simple circular patch; for the highest level of noise, the field largely consisted of ephemeral, local peaks. Measured light levels at the surface of the tank ranged from 4.2 lux (approximately twilight) to 150 lux (overcast day), corresponding to their natural environment in the morning or evening. We stress, however, that light is used as a proxy for any important environmental cue (such as temperature or salinity), with the circular patch representing large-scale features and the noise recreating fine-scale structure.

Because golden shiners are highly cryptic when in dark regions, the tank was lit with infrared light. The level of the projected light field, with respect to their positions, was then used to calculate a 
Fig. 1. (A) The positions of a group of 32 fish as they negotiate the light field, superimposed on that field. Medium noise level $(0.25)$ is shown. Note that the frame only includes a small region of the larger tank. The snapshots are $2 \mathrm{~s}$ apart, with time increasing to the right. (B) Performance, $\Psi$, as a function of group size. The data points show the mean $\Psi$ over experimental trials, and the error bars show twice the standard error. The solid blue line is the statistical model's fit to the data, and the shaded region is its $95 \%$ confidence interval. The red line is the equivalent perform-

A

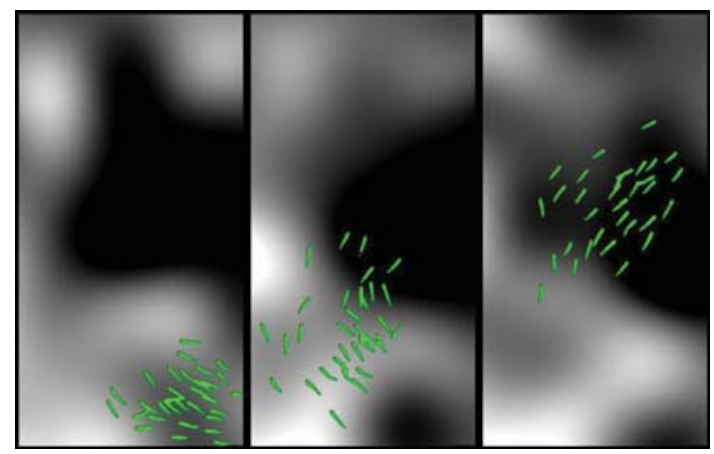

B

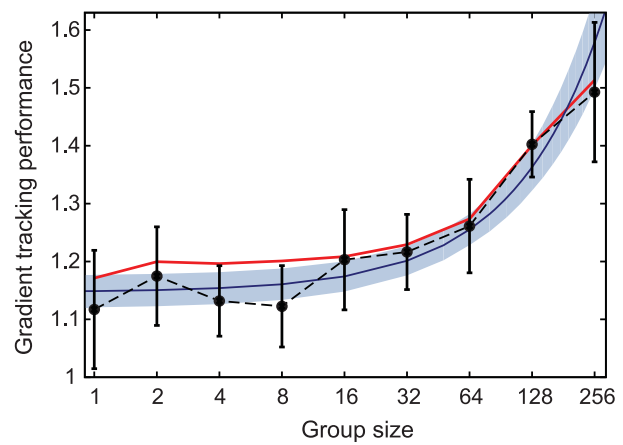

ance measure for a numerical schooling model that incorporates a simple speed-differential algorithm. The quantitative values of this curve are dependent on model parameters, but the qualitative trend is independent of implementation details (16).

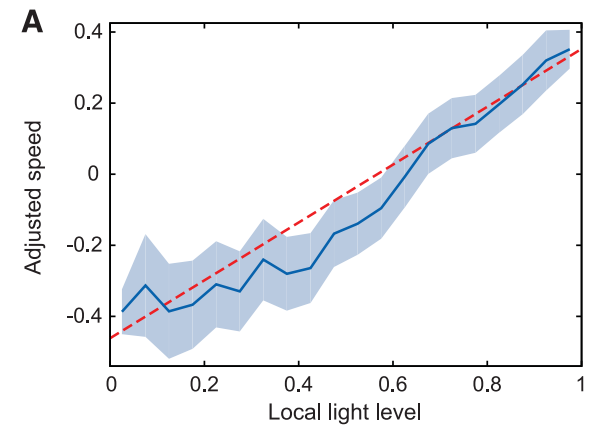

Fig. 2. (A) Average speed as a function of local light level (solid blue curve; 0 is darker, 1 is brighter). Speeds are control-adjusted to account for spatial effects induced by tank walls. The dashed red line is the median slope of the speed-light relation when calculated at single points in time across the group, averaged over group sizes 16 to 128 (slopes at instantaneous time could not be calculated reliably for group sizes of eight and below). (B) Individual response to social and environmental cues as a function of the magnitude of the social vector, $|\mathbf{S}|$. Solid and
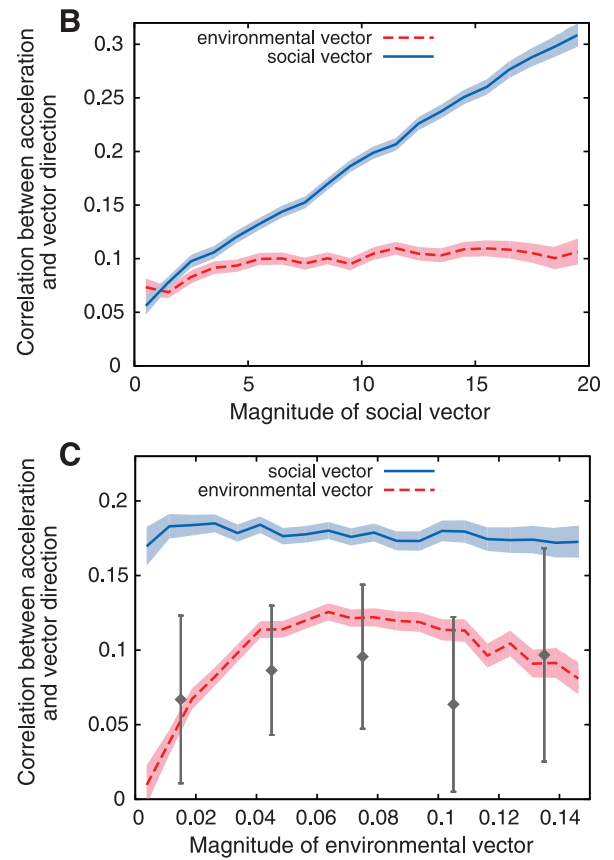

dashed lines show the correlation between the direction of acceleration and the direction of the social vector and environmental vector, respectively. (C) Individual response to social and environmental cues as a function of the magnitude of the environmental vector, $|\mathbf{G}|$. Legend as (B). Gray diamonds show solitary fish and demonstrate that the response to the environmental vector is approximately the same for individuals and those in groups. All shaded regions and error bars denote twice the standard error, data in (A) is down-sampled to remove any spatial correlations in speed and light level. See (16) for details and group sizes shown separately.

performance metric, $\Psi$ : the time-averaged darkness level at the locations of the fish. This was normalized by a null performance value such that $\Psi=1$ in the absence of an environmental response. The value of $\Psi$ increases above unity as performance increases, up to a theoretical maximum of $\Psi_{\max }=3.2 \pm 0.5(16)$.

The level of performance, $\Psi$, increased greatly as a function of group size (Fig. 1B). The value of $\Psi$ was marginally above 1 for the smallest group sizes and for single fish; thus, fish always sought darker regions, regardless of group size. However, larger groups were significantly more effective at doing so $\left[\chi^{2}{ }_{1}=67, P=3.3 \times 10^{-16}\right.$, linear mixed model (LMM)]. Results were not dependent on the level of noise in the projected light field (noise main effect: $\chi_{1}^{2}=0.016, P=0.97$, noise $\times$ group size: $\chi_{1}^{2}=0, P=1, \mathrm{LMM}$ ). The strategy is therefore robust, as it is unaffected by the structure and complexity of the light field.

In order to investigate the mechanism underlying the observed collective response to light gradients, fish were tracked individually to obtain trajectories (16). For all group sizes, we found a strong positive relation between individual speed and the light level at the location of that fish; fish tended to travel more slowly in dark regions and more quickly in light regions, as shown in Fig. 2A.
Using the accelerations of each fish, we assessed the degree to which the motion of individuals may be explained by environmental and social influences. On the basis of attraction to conspecifics, we estimated a social vector, $\mathbf{S}_{i}$, for each individual, $i$, by taking into account the positions of neighbors within a fixed distance. We used seven body lengths, or $34.3 \mathrm{~cm}$, for this distance; however, results are not dependent on the specific range (16). In addition, we measured the environmental vector, $\mathbf{G}_{i}$, for each fish, which points in the direction of steepest ascent in the darkness level with a magnitude proportional to the rate of increase (the gradient of the light field measured from the perspective of the focal fish). We then measured the actual response of individual fish to these potential cues $\left(\mathbf{S}_{i}\right.$ and $\left.\mathbf{G}_{i}\right)$ as the correlation between the direction of these influences and the direction of their acceleration (16).

The relation between the predicted influence ( $x$ axis) and the observed response ( $y$ axis) to $\mathbf{S}_{i}$ and $\mathbf{G}_{i}$, respectively, is shown in Fig. 2, B and C. Acceleration was much more strongly influenced by the location of conspecifics than by environmental gradients (Fig. 2, B and C). When the magnitude of the social vector was high (nearneighbors were located in a consistent direction from the focal individual), the social influence was entirely dominant (Fig. 2B). Groups and solitary fish responded similarly to gradients (Fig. $2 \mathrm{C})$. The slight dip in the response of groups to very high gradients can be attributed to the fact that such steep changes in light level are contributed predominantly by local, ephemeral noise (fig. S17), not by the longer-range feature to be tracked.

Although there is an interdependence between social and environmental effects, because of the increased likelihood of a fish being located in darker regions, these results show that the location of near-neighbors is a far better predictor of an individual's motion than the direction of the nearest dark region. Thus, when making movement decisions, fish more strongly respond to social cues than they do to the environment.

We isolate two complementary processes that result in effective gradient tracking by larger groups. 
To illustrate the first mechanism, consider a scenario where a polarized group is travelling perpendicular to a gradient in light level. Individuals within the group, if located on the brighter side of the environment, will travel more quickly. This, in conjunction with interindividual attraction, induces rotation and turning toward the darker region. We find that, for a single instance in time, variation in speed among fish within a group corresponds to the variation in light level at their locations (Fig. 2A, dashed line). This speed differential across a group causes turning toward those who move more slowly (fig. S10). Second, as individuals slow down, the distance between them is reduced (see fig. S14), which increases the local density of animals in darker regions. Social influence results in acceleration tending to be in this direction (Fig. 2B).

To demonstrate the generic nature of these results, we performed simulations of grouping individuals $(17,18)$ that moderate their speed according to their local light level. Simulated individuals move within the same light fields that were presented to the real fish, yet lack any explicit gradient detection capacity. As in the experiments, greater group-level responsiveness to the environment arises spontaneously as group size increases (Fig. 1B). Further, we show that, although increased group numbers reduce measurement error, the key determinant of performance is the spatial extent of the group in relation to the length scale of the environment; groups that span a larger area are more likely to capture the variation in cue required to elicit a speed differential across the group (16).

Motion toward darker regions (taxis), therefore, results from social interactions among individuals, each of whom exhibits a rudimentary, nondirectional response to their environment (kinesis). Thus, the collective dynamics create a group-level responsiveness to the environment that is absent at the individual level (19). The resulting increase in gradient-tracking ability for larger groups agrees with previous hypotheses $(20,21)$ and could explain empirical studies showing that grouping facilitates the detection of chemical cues (22-24) and improves the accuracy of migrations that rely on such cues $(25,26)$.

The simple algorithm revealed here may allow groups to respond to environmental gradients that occur over long length scales, for instance during the seasonal migration of fish tracking a single isotherm (27). If the mechanism we observed here is found to be widespread, as would be suggested by its robust nature and ease of implementation (in evolutionary terms), there are important ramifications for ecosystem conservation and management. Our results demonstrate that the ability to respond to environmental information may decline as populations are fragmented and average group sizes decrease.

\section{References and Notes}

1. A. W. Woolley, C. F. Chabris, A. Pentland, N. Hashmi, T. W. Malone, Science 330, 686 (2010).

2. D. Grünbaum, Evol. Ecol. 12, 503 (1998).

3. A. M. Simons, Trends Ecol. Evol. 19, 453 (2004).

4. E. A. Codling, J. W. Pitchford, S. D. Simpson, Ecology 88, 1864 (2007).

5. P. A. Larkin, A. Walton, J. Fish. Res. Board Can. 26, 1372 (1969).

6. C. W. Clark, M. Mangel, Theor. Popul. Biol. 30, 45 (1986).

7. I. D. Couzin et al., Science 334, 1578 (2011).

8. N. R. Franks, S. C. Pratt, E. B. Mallon, N. F. Britton, D. ]. Sumpter, Phil. Trans. R. Soc. B 357, 1567 (2002). 9. F. Galton, Nature 75, 450 (1907).

10. S. Bauer et al., in Animal Migration: A Synthesis, E. J. Milner-Gulland, ]. M. Fryxell, A. R. E. Sinclair, Eds. (Oxford Univ. Press, New York, 2011), pp. 68-87.

11. T. J. Pitcher, ]. K. Parrish, Behaviour of Teleost Fishes (Springer, New York, 1993), vol. 2, pp. 369-439.

12. R. Poli, ]. Kennedy, T. Blackwell, Swarm Intell. 1, 33 (2007).

13. P. Ogren, E. Fiorelli, N. E. Leonard, IEEE Trans. Automat. Contr. 49, 1292 (2004).
14. S. G. Reebs, Anim. Behav. 59, 403 (2000).

15. D. J. Hall et al., J. Fish. Board Can. 36, 1029 (1979).

16. Materials and methods are available as supplementary materials on Science Online.

17. I. D. Couzin, ]. Krause, N. R. Franks, S. A. Levin, Nature 433, 513 (2005).

18. M. Camperi, A. Cavagna, I. Giardina, G. Parisi, E. Silvestri, Interface Focus 2, 715 (2012).

19. C. J. Torney, Z. Neufeld, I. D. Couzin, Proc. Natl. Acad. Sci. U.S.A. 106, 22055 (2009).

20. A. Huth, C. Wissel, Ecol. Modell. 75-76, 135 (1994).

21. U. Kils, thesis, University of Kiel, Germany (1987).

22. R. E. McNicol, E. Scherer, J. H. Gee, Environ. Biol. Fishes 47, 311 (1996).

23. L. W. Hall Jr., D. T. Burton, S. L. Margrey, W. C. Graves, J. Toxicol. Environ. Health 10, 1017 (1982).

24. C. W. Steele, A. D. Scarfe, D. W. Owens, J. Fish Biol. 38, 553 (1991).

25. T. P. Quinn, K. Fresh, Can. J. Fish. Aquat. Sci. 41, 1078 (1984).

26. J. J. Hard, W. R. Heard, Can. J. Fish. Aquat. Sci. 56, 578 (1999).

27. K. Schaefer, D. Fuller, B. Block, Mar. Biol. 152, 503 (2007)

Acknowledgments: We thank ]. Herbert-Read for experimental assistance, N. O. Handegard for providing code and advice for track linking, and A. Haenssen for technical support. Suggestions from members of the Couzin laboratory and two anonymous reviewers greatly improved this manuscript. Funded by NSF awards PHY-0848755, EAGER-1251585, and CNH-1211972; U.S. Office of Naval Research award N00014-09-1-1074; U.S. Army Research Office grant W911NG-11-1-0385; Leverhulme Early Career Fellowship; Natural Sciences and Engineering Research Council of Canada Postgraduate Scholarship Fellowship; and the Yukon Foundation. All experiments were conducted in accordance with federal and state regulations and were approved by the Princeton University Institutional Animal Care and Use Committee. Code freely available at http://icouzin.princeton.edu. 